Article

\title{
Implementing Cancer Care in Rwanda: Capacity Building for Treatment and Scale-Up
}

\author{
Paul H. Park ${ }^{1,2,3, *(1)}$, Cyprien Shyirambere ${ }^{4}$, Fred Kateera ${ }^{4}$, Neil Gupta ${ }^{1,2,3}$, Christian Rusangwa ${ }^{4}$, \\ Joia Mukherjee ${ }^{1,2,3}$, Alex Coutinho ${ }^{4}$, Leslie Lehmann ${ }^{3,5}$, Lori Buswell ${ }^{5}$, Lawrence N. Shulman ${ }^{6}$, \\ Joel M. Mubiligi ${ }^{4}$, Francois Uwinkindi ${ }^{7,+}$ and Lisa R. Hirschhorn ${ }^{8,+}+$
}

check for updates

Citation: Park, P.H.; Shyirambere, C.; Kateera, F.; Gupta, N.; Rusangwa, C.; Mukherjee, J.; Coutinho, A.;

Lehmann, L.; Buswell, L.; Shulman,

L.N.; et al. Implementing Cancer Care in Rwanda: Capacity Building for Treatment and Scale-Up. Sustainability 2021, 13, 7216. https:// doi.org/10.3390/su13137216

Academic Editor: Giuseppe Battaglia

Received: 8 March 2021

Accepted: 23 June 2021

Published: 28 June 2021

Publisher's Note: MDPI stays neutral with regard to jurisdictional claims in published maps and institutional affiliations.

Copyright: (c) 2021 by the authors. Licensee MDPI, Basel, Switzerland. This article is an open access article distributed under the terms and conditions of the Creative Commons Attribution (CC BY) license (https:/ / creativecommons.org/licenses/by/ $4.0 /)$.
1 Partners in Health, Boston, MA 02199, USA; ngupta@pih.org (N.G.); jmukherjee@pih.org (J.M.)

2 Department of Medicine, Division of Global Health Equity, Brigham and Women's Hospital, Boston, MA 02115, USA

3 Harvard Medical School, Boston, MA 02115, USA; Leslie_Lehmann@DFCI.HARVARD.EDU

4 Inshuti Mu Buzima/Partners in Health, Rwinkwavu, Rwanda; cshyirambere@pih.org (C.S.); fkateera@pih.org (F.K.); elchristiano2020@gmail.com (C.R.); agcoutinho@icloud.com (A.C.); jmubiligi@pih.org (J.M.M.)

5 Center for Global Cancer Medicine, Dana-Farber Cancer Institute, Boston, MA 02215, USA; Lbuswell@partners.org

6 Abramson Cancer Center, University of Pennsylvania, Philadelphia, PA 19104, USA; Lawrence.shulman@uphs.upenn.edu

7 Rwanda Biomedical Centre, Division of Non-Communicable Diseases, Kigali, Rwanda; francois.uwinkindi@rbc.gov.rw

8 Department of Medical Social Sciences, Northwestern University Feinberg School of Medicine, Chicago, IL 60611, USA; lisa.hirschhorn@northwestern.edu

* Correspondence: ppark@bwh.harvard.edu

+ Co-senior authors.

Abstract: Background: The majority of countries in sub-Saharan Africa are ill-prepared to address the rising burden of cancer. While some have been able to establish a single cancer referral center, few have been able to scale-up services nationally towards universal health coverage. The literature lacks a step-wise implementation approach for resource-limited countries to move beyond a singlefacility implementation strategy and implement a national cancer strategy to expand effective coverage. Methods: We applied an implementation science framework, which describes a fourphase approach: Exploration, Preparation, Implementation, and Sustainment (EPIS). Through this framework, we describe Rwanda's approach to establish not just a single cancer center, but a national cancer program. Results: By applying EPIS to Rwanda's implementation approach, we analyzed and identified the implementation strategies and factors, which informed processes of each phase to establish foundational cancer delivery components, including trained staff, diagnostic technology, essential medicines, and medical informatics. These cancer delivery components allowed for the implementation of Rwanda's first cancer center, while simultaneously serving as the nidus for capacity building of foundational components for future cancer centers. Conclusion: This "progressive scaling" approach ensured that initial investments in the country's first cancer center was a step toward establishing future cancer centers in the country.

Keywords: universal health care; cancer; implementation science; sub-Saharan Africa; Rwanda; universal health coverage; sustainable development goals

\section{Introduction}

The rising burden of cancer in low and middle-income countries (LMICs) is welldocumented, as these countries encounter growing and aging populations and changing epidemiology of major burdens of disease [1,2]. Despite $70 \%$ of new cancer cases globally projected to occur in LMICs by 2030 , only $5 \%$ of global cancer resources are dedicated 
to these countries [3]. In sub-Saharan Africa, only 11 of 32 countries had national cancer control plans as of 2013 [4]. This translates into many sub-Saharan African countries having established none or only a single public cancer treatment center with no additional public facilities able to provide treatment. Even in countries with a national referral center, travel across long distances to a single referral hospital for treatment courses and routine follow-up visits are barriers for many patients. Without fully trained and equipped services scaled beyond a single referral center, the rising cancer burden will continue without an effective treatment response. The global focus on universal health coverage (UHC) and the Sustainable Development Goals (SDGs) regarding health care for all, equality, and the means of implementation suggest that population-level cancer care should be a priority. However, current implementation approaches are falling short of this goal by placing too much of the care delivery burden on single cancer referral centers without plans for scale-up.

While there are a growing number of studies focused on how to scale and sustain care delivery programs for other diseases, in cancer, there remains a large knowledge gap in the published literature on how to support the implementation and scaling of cancer programs $[5,6]$. This challenge is in part due to the wide range of cancers and complex diagnoses and need for multi-modality treatment. Without this critical knowledge on implementation and sustainability, LMICs will continue to struggle to establish and scale effective evidence-based cancer programs. Even once a country establishes its first cancer center, identifying and implementing strategies to effectively scale and sustain cancer facilities in their country is needed. Implementation science (IS) is the study of methods to assess the quality and outcomes and promote the implementation of evidence-based practices, interventions, and policies into routine health care [7]. The methodology offers a growing knowledge base of frameworks and implementation strategies, and outcomes can inform planning, implementing, sustaining, and scaling delivery systems in cancer and other areas. IS frameworks can guide national programs planning for immediate implementation and future scale. This includes identifying existing strengths and gaps to inform effective implementation strategies, which can simultaneously establish much needed services as well as plan for spread through building foundational components needed for future scale-up. IS frameworks can also inform an evaluation approach, which can produce the knowledge needed for local and global improvement, scale, and sustainability.

Aarons et al. describes a four-phase model of implementation: Exploration, Preparation, Implementation, and Sustainment (EPIS) [8]. The EPIS framework focuses on identifying key strategies, which drive each of the four phases, and existing contextual factors that help to plan for and extract understanding of the success and challenges of the sustainable implementation of evidence-based approaches to health systems. Contextual factors include characteristics of the health system itself (internal) or outside polices and partners (external) that have a direct impact on facilitating or inhibiting the four phases of EPIS and targeted implementation outcomes. The EPIS framework has been used to evaluate public health and health care delivery implementation projects globally, including LMICs [9]. For example, in Botswana, Tapela and colleagues applied the EPIS framework to describe the government strategies to integrate noncommunicable disease care, including cancer services, into primary health care [10]. Another study by Williams and colleagues applied EPIS by examining the impact of organizational characteristics and strategies on the adoption of a novel care delivery approach for community health organizations [11].

The aim of this work is to apply EPIS to examine and identify the implementation process undertaken by the Rwanda Ministry of Health (RMOH) to establish not only its first national cancer center, but also the foundational components needed to scale a national cancer program towards achieving equitable access to comprehensive care and UHC.

\section{Materials and Methods}

The implementation of the Butaro Cancer Center of Excellence (BCCOE) is welldocumented as a successful first national cancer facility for Rwanda [12-18]. In this paper 
we retrospectively apply the EPIS framework to better understand the implementation of a national cancer program. The areas of implementation covered include both cancer care at BCCOE and capacity building for the foundational cancer care delivery components needed to support the scale-up of cancer care and establishment of future cancer centers in Rwanda. We have termed this step-wise approach by the Rwanda Ministry of Health $(\mathrm{RMOH})$ for expanding effective cancer care coverage to achieve national coverage as "progressive scaling". Progressive scaling is a potentially resource-efficient strategy to build on existing referral centers and close the global health delivery gap in cancer care, especially in low-income countries (LICs).

Data sources included existing government reports, peer-reviewed literature from the BCCOE, programmatic documents from implementation partners, Partners In Health (PIH), Dana-Farber Cancer Institute (DFCI), Brigham and Women's Hospital (BWH), and Harvard Medical School (HMS). One author (PHP) extracted information on key contextual factors and implementation strategies in each of the four EPIS stages. The results were reviewed and expanded through discussion with the co-authors who were directly involved in the exploration, preparation, implementation, and building for sustainment.

\section{Results}

Upon applying EPIS to the Rwanda national cancer program, we identified the following strategies to scale cancer care delivery components in each of the four phases.

\subsection{Exploration Phase}

The exploration phase of implementing a national cancer program began with the identification of the severe limitation of cancer services and unaddressed burden of disease in Rwanda. With the support of PIH-Rwanda, locally known as Inshuti Mu Buzima (IMB), and oncology technical experts from large academic tertiary medical centers, DFCI and $\mathrm{BWH}, \mathrm{RMOH}$ completed a situational analysis to identify the key gaps in cancer care delivery. Prior to 2012, cancer care in Rwanda existed only at a few referral hospitals, which had no oncologist or trained oncology nurse, limited pathology services and medication formulary, only basic surgery, and high costs of care. Securing a reliable treatment option typically required transfer of the patient to another country [12-14]. As BCCOE was a new center at Butaro District Hospital, there were no existing formal oncology care resources, such as staff or equipment, prior to the center's implementation. Such gaps highlighted the need for broad external support to start the implementation, especially technical expertise.

Rwanda recognized the need to establish a new approach to domestic cancer care by implementing its first cancer center. The Butaro District Hospital was established by the $\mathrm{RMOH}$ in 2011 with support from IMB, who has been working with RMOH since 2005. As a key contextual factor, IMB had already established itself as a key partner to the RMOH by co-developing novel, evidence-based care delivery programs that could be implemented and assessed for potential scale-up [19-21]. This culture provided a strong absorptive capacity and readiness for change when it came to establishing a new cancer center with potential for future scale-up (see Table 1). Likewise, the decision to place the BCCOE at a rural district hospital supported by IMB in the Northern Province was driven by the RMOH's desire to identify a site to test and adapt approaches in a smaller scale environment that would ultimately serve as a nidus for national scale-up.

\subsection{Preparation Phase}

Aarons et al. highlights academic-public partnership and evidence-based care as two key factors which are helpful in the preparation phase [8]. From the beginning of preparation for a national cancer program, RMOH actively sought the expert opinion of implementation and academic partners, including IMB and DFCI. These partners helped to ensure development of standards of cancer care appropriate for Rwanda, supported the design of contextually appropriate innovative solutions to care delivery, and provided initial on-site implementation support across the broad scope of foundational building 
blocks required for a cancer care program while jumpstarting care delivery. The RMOH identified the following foundational components needed for cancer care delivery: staff (oncology-trained nurses, physicians, laboratory technicians), clinical care protocols, pathology, essential medicines, surgery, radiotherapy, socioeconomic support, medical record system, monitoring and evaluation, and research. Together with stakeholders, the RMOH defined a basic minimum package of these foundational components required to open a cancer center. This package included clinical and pathology laboratory services with trained technicians and standard safety features, essential medicines for selected priority cancers, nurses trained in standard operating procedures (SOPs) for chemotherapy preparation and administration with safety measures in place, and essential radiology technology. The specific design and selection of these components were based on a formal and rigorous process of prioritization inclusive of consultation with technical experts as well as consideration of the context of the facility. The RMOH guided significant upfront support from academic partners to ensure that a strong methodologic approach to building facility and human resource capacity could establish a strong precedence of quality cancer care, which would eventually be primed for scale-up and sustainability.

Table 1. EPIS-based implementation strategies for establishing BCCOE as a center for progressive scaling to support a national cancer care program in Rwanda.

\begin{tabular}{|c|c|c|}
\hline Implementation Strategies & Description & Contextual Factors \\
\hline \multicolumn{3}{|c|}{ Exploration Phase } \\
\hline Situational analysis and priority setting & $\begin{array}{l}\text { - Situational analysis to identify gaps } \\
\text { in cancer care: Observation, } \\
\text { assessment, and acknowledgement } \\
\text { of gaps in cancer care across the } \\
\text { country } \\
\text { Priority setting through national } \\
\text { policies to support establishment of } \\
\text { national cancer center as the first } \\
\text { step to progressive scaling }\end{array}$ & $\begin{array}{l}\text { Internal: Severe budget restrictions } \\
\text { had previously limited growth of } \\
\text { cancer program; Smaller scale } \\
\text { facilities identified as having high } \\
\text { absorptive capacity }\end{array}$ \\
\hline Stakeholder engagement & $\begin{array}{l}\text { Technical working group meetings } \\
\text { to explore the gaps in care and } \\
\text { potential solutions }\end{array}$ & $\begin{array}{l}\text { External: Potential partners ready to } \\
\text { provide technical support, funding, } \\
\text { and other essential resources for } \\
\text { cancer care and building capacity }\end{array}$ \\
\hline \multicolumn{3}{|c|}{ Preparation Phase } \\
\hline Define priorities and goals & $\begin{array}{l}\text { Define priorities and goals for } \\
\text { starting a national cancer program; } \\
\text { planning areas include: human } \\
\text { resources, procurement of } \\
\text { medicines and equipment, } \\
\text { partnerships for technical expertise } \\
\text { and other resources, and physical } \\
\text { infrastructure; led by MOH } \\
\text { Progressive scaling: The newly } \\
\text { established infrastructure of BCCOE } \\
\text { served as a platform from which } \\
\text { care delivery components for future } \\
\text { cancer centers is built }\end{array}$ & $\begin{array}{l}\text { Internal: existing national health } \\
\text { strategy is supportive of } \\
\text { establishment of national cancer } \\
\text { program; organizational culture and } \\
\text { values of MOH and partners are } \\
\text { receptive to taking on new program }\end{array}$ \\
\hline
\end{tabular}


Table 1. Cont.

\begin{tabular}{|c|c|c|}
\hline Implementation Strategies & Description & Contextual Factors \\
\hline Evidence-based approach & $\begin{array}{l}\text { Review of evidence-based } \\
\text { interventions and adaptation to } \\
\text { local setting } \\
\text { Execution and review of baseline } \\
\text { assessment studies as to describe } \\
\text { contextual factors. These include } \\
\text { system readiness surveys and } \\
\text { qualitative interviews of providers } \\
\text { and patients }\end{array}$ & $\begin{array}{l}\text { Internal: } \mathrm{MOH} \text { provided existing } \\
\text { culture of data use and research to } \\
\text { inform planning and policies }\end{array}$ \\
\hline Stakeholder engagement & $\begin{array}{l}\text { Technical expertise: Partners filled } \\
\text { the role of intervention } \\
\text { co-developers; examples included } \\
\text { clinical care delivery work flow, } \\
\text { clinical protocols, and training } \\
\text { curricula and tools }\end{array}$ & $\begin{array}{l}\text { External: Precedence of large } \\
\text { subsidization of essential resources } \\
\text { by international partners to launch } \\
\text { national care programs; expectation } \\
\text { of continued precedence for } \\
\text { initiating cancer care; } \\
\text { Internal: RMOH providing staffing } \\
\text { and other infrastructure resources } \\
\text { with the long term plan of RMOH } \\
\text { leading the financing of all clinical } \\
\text { programs }\end{array}$ \\
\hline \multicolumn{3}{|c|}{ Implementation Phase } \\
\hline Building on existing infrastructure & $\begin{array}{l}\text { BCCOE serves as nidus for national } \\
\text { cancer program: Establishment of } \\
\text { cancer foundational components } \\
\text { would serve the immediate needs as } \\
\text { well as capacity building for future } \\
\text { cancer facilities }\end{array}$ & $\begin{array}{l}\text { - Internal: Butaro District Hospital } \\
\text { had existing human resources, } \\
\text { space, equipment, and leadership } \\
\text { structure which could provide a } \\
\text { starting point from which the } \\
\text { BCCOE cancer delivery components } \\
\text { could be built alongside } \\
\text { international partners } \\
\text { External: Commitment of Rwanda } \\
\text { Military Hospital and other } \\
\text { hospitals to gain expertise and } \\
\text { knowledge by sending nurses to } \\
\text { BCCOE for training. }\end{array}$ \\
\hline
\end{tabular}

- Broad government involvement and stakeholder alignment included Central MOH Noncommunicable disease division, Cancer program, Butaro District Hospital leadership, and BCCOE leadership

$\mathrm{MOH}$ leadership

- Implementation partners work at central and local levels to better understand the needs, challenges, and successes

- Regular progress report meetings to discuss success and challenges, review available data, and plan strategies for advancement
- Internal: Centralized leadership structure; culture of openness to innovation and change 
Table 1. Cont.

\begin{tabular}{|c|c|c|}
\hline Implementation Strategies & Description & Contextual Factors \\
\hline Resources for implementation & $\begin{array}{l}\text { - Advocacy for continuation of } \\
\text { policies and political will, which } \\
\text { support resource allocation towards } \\
\text { national cancer program } \\
\text { Essential care delivery resources: } \\
\text { Initial high dependence on external } \\
\text { support } \\
\text { Essential administrative resources } \\
\text { included meetings, training, } \\
\text { computer systems, office } \\
\text { consumables, and others }\end{array}$ & $\begin{array}{l}\text { Internal: Limited financial and } \\
\text { human resources to implement care } \\
\text { delivery }\end{array}$ \\
\hline
\end{tabular}

Sustainment Phase

- Maintain prioritization of cancer care delivery through renewal and/or expansion of existing cancer-related policies; include cancer in UHC

Maintain and expand policy and funding support

- Financial responsibility:

Establishment of a clear plan for stepwise transition of financial responsibility led by $\mathrm{MOH}$
- $\quad$ External: Strong climate of interest in cancer care among potential and existing academic and private partners

- Internal: Limited funds available despite prioritization of cancer care at a policy level
- Human resources incentives in the form of job promotion and capacity building opportunities (education courses, conferences, workshops, etc.)

Staff retention and expansion

Fidelity, adoption and feasibility monitoring
- Retention of experienced clinical and program staff leads to sustained and/or improved quality at the facility as well as strong training of visiting clinicians/trainees for future cancer centers
- $\quad$ External: Ministry of Education and University of Rwanda have prioritized cancer education among nurses through establishment of master's in oncology nursing program
- Designing and implementing data collection tools for monitoring and evaluation of clinical presentations, clinical outcomes, and adherence to SOPs and clinical protocols

- $\quad$ Routine data evaluation informs discussions and subsequent policy decisions and prioritization for quality improvement and sustainment projects
- Internal: $\mathrm{MOH}$ has strong culture of prioritizing monitoring and evaluation for policy and quality improvement purposes

- $\quad$ External: Academic partners have a strong interest in data evaluation and research

The foundational components prioritized essentialness and quality of services to be delivered at the center, but were also designed to support planned progressive scaling. Progressive scaling meant that the newly established foundational components within the BCCOE would be designed to also be platforms for capacity building and produce replicable cancer care delivery components for future cancer centers in Rwanda. For example, it was planned that expert cancer nurses working in the BCCOE would also serve as trainers to educate cancer nurses for other planned cancer centers. This progressive scaling approach would allow investments in BCCOE to generate benefits for not only the 
single facility itself and the patients it served, but also scale cancer care to new facilities across Rwanda.

Due to the limited financial resources in-country, the initial financing mechanism for the national cancer program relied heavily on support from the strategic partners, additional foundations, and private donors. Reflecting prior collaborative projects within the PIH-RMOH partnership designed for scale and sustainability [19-21], the long-term sustainment plan was for the $\mathrm{RMOH}$ to progressively assume the financing of the $\mathrm{BCCOE}$, including its role as a national training center for downstream scale-up of cancer services to other facilities. This transition to RMOH-led financing was recognized to require significant time and a strategic approach as discussed below (see Table 1).

\subsection{Implementation Phase}

As planned, the implementation of the national cancer program began with the development of the targeted foundational components of cancer care at BCCOE meant to facilitate scale through: (1) designing components for replication (e.g., clinical protocols, electronic medical records); (2) technical training at the center; and (3) organizational and implementation planning support to staff at new cancer care facilities. The basic components were implemented during a one-year period. BCCOE based in an expanded district hospital functioned as the national referral cancer center and provided a broad spectrum of services including initial evaluation, biopsy, pathology, staging, outpatient and inpatient chemotherapy, surgery, international radiotherapy referrals, palliative care, mental health and psychological support, community-based early detection, social support, and patient follow-up through an electronic medical record system (OpenMRS) [12,14]. Throughout the process, the RMOH, IMB, DFCI, and other partners maintained clear roles and responsibilities of collaboration at both the central policy and local implementation levels as to develop these services to produce the foundational components for future facilities. The strategies used in the Implementation Phase answered a need for immediate capacity building for cancer services in Butaro, but also for future cancer centers across Rwanda (see Table 1).

A key strategy for establishing a national cancer program was developing and planning for building on existing infrastructure. The early established infrastructure at BCCOE provided a strong platform from which additional care delivery components for itself and future cancer centers were built. There are several examples that follow which describe how implementation at BCCOE led to progressive scaling for the national cancer program.

\subsubsection{Human Resources: Oncology Nurses and Physicians}

To support MOH clinical capacity, DFCI has seconded trained US oncology nurses to BCCOE nearly continuously since March 2012 in order to develop capacity at the site but also plan for scale. These specialized nurse trainers supported the development of nursing standards, SOPs and the education of the adult and pediatric nurses at BCCOE. Building on this capacity, BCCOE then conducted nationally certified three-month oncology nurse trainings where nurses from Rwanda's referral hospitals were invited to Butaro and received didactic and practical education and subsequently returned to their respective hospitals to become key members of their first cohort of oncology nurses [22]. BCCOE also provided pre-service training by hosting students from the University of Rwanda Master's in Oncology Nursing program for practical training. University of Rwanda interns and resident physicians in internal medicine, surgery, and pathology also rotated through BCCOE as part of their medical education to gain experience in cancer care. Two such interns at BCCOE would later go on to work at the new cancer center in the Rwanda Military Hospital.

\subsubsection{National Cancer Clinical Protocols and Oncology Nursing Standard Operating Procedures}

The clinical protocols for cancer treatment and cancer nursing procedures established and tested at BCCOE served as the national standards. RMOH led a technical working 
group, which continuously updated these protocols with the support of BCCOE staff, IMB, DFCI, and Rwandan physicians from newly established cancer referral hospitals. The protocols have been readily available and disseminated and implemented in two other facilities treating cancer in Rwanda.

\subsubsection{Cancer Medical Records}

As another key contextual factor, the RMOH had previously identified OpenMRS as its national EMR platform for all disease areas, and therefore BCCOE's oncology OpenMRS EMR platform was well-positioned to support and guide EMR implementation at other public facilities. As to set a strong precedent for quality EMR at BCCOE and future cancer centers, the oncology OpenMRS platform incorporated key safety features, including computerized chemotherapy order entry templates. Currently, the $\mathrm{MOH}$ is planning to implement the oncology OpenMRS platform at selected referral hospitals.

\subsubsection{Pathology}

BCCOE took a step-wise approach to developing pathology services and capacity starting from basic services to including telepathology and then implementing immunohistochemistry and GeneXpert molecular testing capacity while also training on-site pathologists [23]. The American Society of Clinical Pathology (ASCP) leadership, already familiar with this stepwise progression of pathology capacity, selected BCCOE to be the first site globally to receive the high output and automated technologies necessary to become a regional telepathology center [24]. Building on BCCOE capacity, the BCCOE pathology program has also supported the stepwise progression of pathology programs at subsequent cancer centers in Rwanda and other LICs [25]. Scaling this high output approach at the Rwanda Military Hospital cancer program is already complete.

\subsubsection{Essential Medicines}

In contrast to the components detailed above, the progressive scaling of access to essential cancer medicines does not yet have a clear path forward. At BCCOE, the high costs of chemotherapy agents led IMB, DFCI, and other partners to procure a limited selection of generic cancer medicines and subsidize the cost as a means to ensure affordable access to care in the short term. This approach of utilizing strategic partnerships to financially support such costs is not dissimilar to that of the early HIV programs in LICs [26]. Agreements with several other partners, including Novartis and the Max Foundation, served as first steps in providing significant cost reductions to tamoxifen, letrozole, and imatinib $[27,28]$. The price reduction of these essential medicines not only alleviated part of the financial pressure at BCCOE but also made medicines more accessible at new cancer delivery facilities in Rwanda. The RMOH has also placed essential cancer drugs on their national essential medicines list. As a next step, the $\mathrm{RMOH}$ is negotiating to have these medications added to the list of medicines covered by the national health insurance program, Mutuelle de Sante. If approved, the national health insurance program would be able to cover a substantial portion of the cost to patients.

\subsection{Sustainment Phase}

As the implementation phase of a national cancer program continues to progress, the scope of work has increased focus on sustainability and inclusion of cancer in national UHC goals (see Table 1). Continuing and expanding care at the BCCOE includes specific mechanisms by which BCCOE fulfills its role as a source for progressive scaling to new cancer centers. Two early examples include staff retention and monitoring and evaluation.

Within the BCCOE, maximizing staff retention and facilitating staff replacement are critical to sustaining quality and the leadership and capacity to support planned scale. In response, the BCCOE established nurse education leadership positions, which provided increased salaries as well as opportunities for senior nurse experts to be recognized for their advanced skills and also participate in national meetings and trainings. This intervention 
provided an incentive for oncology nurses to work towards promotion and placed clear responsibility for these staff to train and capacity build their peers and any new replacement staff. By retaining senior nurses, the training of nurses from other facilities in cancer care became possible. As planned, the initial cadre of physicians at BCCOE were primarily expatriates from the US and East Africa. Over time, as Rwandan physicians were trained in cancer protocols the majority of physicians at BCCOE were Rwandan by 2016. By focusing on recruitment and training of Rwandan staff there exists greater opportunities for retention of providers, and increasing availability of trained healthcare workers for the new centers.

Routine, comprehensive data collection via the oncology OpenMRS EMR allowed for the production and regular review of monitoring and evaluation reports as well as research by BCCOE leadership. This data capacity and use not only provided capacity for monitoring clinical outcomes and gaps in care but also an opportunity to prioritize areas of advocacy for local and national policy change in planning for scale. For example, early outcomes studies and costing evaluations $[29,30]$ provided guidance for financial planning and a forum for advocacy to seek further support for the growth and eventual sustainment of the national cancer program. Key outcomes of interest included clinical metrics as well as implementation outcomes, such as feasibility, fidelity, and adoption. For example, early outcomes showed substantial late stage presentation in breast cancer [12,31]. As a result, the RMOH supported an early detection breast cancer project, which utilized community health workers and health center nurses to increase referral rates of patients with suspected breast cancer $[16,17]$. By detecting cancer at an earlier stage, patients are eligible for curative, as opposed to palliative, treatment, which leads to increased chances of survival. This project has since moved beyond the BCCOE catchment area and has been scaled to other districts. Data-driven communication within the RMOH also informed decisions where to allocate resources based on burden of disease, effectiveness of early detection and referral pathways, and challenges surrounding late stage presentation and access to essential medicines.

As the breadth and volume of $\mathrm{BCCOE}$ services grew, the RMOH established additional partnerships with foundations and academic institutions, such as University of Pennsylvania and Dartmouth Hitchcock Medical Center, to further expand the diversity of expertise and other resources, which can support the needs of both BCCOE and new centers expanding through the RMOH's plans for scaling. As noted, the gradual shift towards more $\mathrm{MOH}$ financially-led initiatives, such as inclusion of oncology medication in Mutuelle de Sante, is designed to support this sustainability.

\section{Discussion}

Rwanda successfully established a national cancer center, which is able to provide needed treatment while simultaneously building capacity to advance towards a national cancer program in Rwanda. Application of the EPIS framework provides a format to understand key implementation strategies and contextual factors, which were used to support the approach of progressive scaling to achieve these dual aims of immediate direct care provision and capacity building. The RMOH's strong leadership and strategic partnerships identified and guided the necessary steps to fill the gap in foundational components at BCCOE while also planning for future cancer centers from the start. This national leadership and coordination reduced the risk of duplication and increased the potential for sustainability. Other collaborative approaches in sub-Saharan Africa to address capacity building within cancer care at a larger scale tend to focus on training of staff, including oncologists and oncology nurses [32]. However, they do not aim to build capacity for a broader range of cancer care components, such as EMR platforms, clinical protocols, standard operating procedures, and pathology services needed to expand care at a national scale. Screening, prevention, and early detections programs have also had some success at national levels; however, they oftentimes do not include a treatment strategy [33]. 
The $\mathrm{RMOH}$ is progressing forward in its goal to produce self-sufficient leadership and resources locally at BCCOE, which serves to support cancer delivery components for future cancer sites. While other national cancer centers operating in Rwanda at the same capacity as BCCOE have not yet been established, the success of the progressive scaling strategy in Rwanda so far has resulted in support of early stage implementation of two other cancer centers with essential staff, clinical protocols, and other cancer care components, such that new cancer centers can begin building from a more advantageous starting point. However, there are cancer delivery components that are not easily addressed by the progressive scaling approach. In particular, access to essential medicines, oncologists, and psychosocial support are not easily scaled. However, $\mathrm{RMOH}$ and its partners have identified strategies, such as establishing in-country master's programs and private-public partnerships, which can lead to increased national-level oncology staffing and access for specific medications, as done for tamoxifen and letrozole in Rwanda [27]. As another example, the Rwanda Military Hospital successfully established partnerships leading to radiotherapy services covered by Mutuelle de Sante [34]. Another strategy employed was task shifting to general practitioners, internists, and pediatricians as an interim measure until more oncologists are trained [35].

The RMOH's strategy of partnership engagement included some key approaches: partnerships are invested in long term, continuous commitment, inclusive of on-the-ground support and are coordinated through RMOH leadership. For example, DFCI not only committed to provide the necessary expertise through frequent in-country visits for stakeholder meetings and weekly tumor boards, but also has continued to support nurse and physician didactic and practical training for nearly a decade with some staff staying on-site for up to a year. Additionally, given IMB's direct presence in rural districts since 2005, IMB staff members observed and experienced the challenges simultaneously alongside their $\mathrm{RMOH}$ colleagues. From this shared vantage point, the RMOH and partners are able to effectively collaborate to identify and execute direct implementation solutions as well as progressive scaling.

While there has been short-term success in producing and sustaining a number of the targeted care delivery components for BCCOE and capacity building for future cancer centers, progress is still needed in several areas, including the substantial dependence on external funding. Moving forward, the growth and sustainment of a national cancer program will require strong government leadership to build upon current policies, which prioritize adequate resource allocation towards cancer care delivery inclusive of a progressive scaling approach. Furthermore local and international advocacy for cost reduction of essential medicines and other technologies will be vital. The establishment of a cancer registry will provide a valuable resource to make data-driven policy decisions surrounding disease prioritization and resource allocation. Future threats to sustainability include poor staff retention, partnership turnover, and poor fidelity monitoring and support. In order to overcome these threats, there is a need for a long-term vision when assessing the time and resources required to produce desired outputs of progressive scaling. The first HIV treatment programs in sub-Saharan Africa were largely viewed as too costly and "unsustainable" according to some definitions [36,37]. Engebretsen, et al. point out that the partnership criteria of requiring a short-term strategy of "sustainability" for the world's poorest regions is a false ideal in that it immediately excludes the most vulnerable populations [38]. While the long-term goal of governments is to become self-sufficient in delivering UHC, strategic, rights-based upfront investments by partners as directed by the government are essential to achieve such goals. As proof-of-concept implementation supports the ongoing advocacy of equitable access to cancer services, costs may continue to decline [39]. Simultaneously, as governments continue to strengthen over time, they will be in a better position to lead the financing effort, especially if costs are reduced. Progressive scaling can serve as one efficient approach to reach self-sufficiency sooner.

There were a number of limitations to our analysis, including the retrospective nature of the application of EPIS. Prospective and methodical evaluation of the EPIS phases with 
clear documentation would have provided more in-depth details for each stage. Additionally, it is too early in the implementation and sustainment phases to have quantitative data surrounding clinical and implementation outcomes of the progressive scaling work and this analysis was done early in the scale up. Future studies of progressive scaling should include baseline as well as follow-up data on key implementation outcomes, such as adoption, feasibility, fidelity and reach of care. Additionally, studies should focus on measuring the success of progressive scaling of cancer care and analyzing the strategies and resources needed to both plan for and implement while also addressing critical needs for existing cancer patients.

Establishing cancer treatment and training centers in LICs is essential if we are to achieve UHC. However, achieving this goal requires that the national strategy incorporates building capacity for national scale-up capacity building, while starting to meet the growing demands for cancer diagnosis and treatment and providing opportunities to learn from local experience. Equitable and effective coverage will require that all cancer patients are able to geographically access and afford quality care while avoiding financial catastrophe and completing the planned treatment. SDGs calls for us to strengthen the means of implementation. The EPIS framework has provided an approach from which strategies and experiences from the implementation of Rwanda's national cancer program can be identified and transformed into actionable knowledge to further inform scale-up in Rwanda and other countries aiming to ensure access to cancer care.

\section{Conclusions}

Although the implementation challenges are complex and reflect both shared and local contextual factors, progressive scaling is an approach that can facilitate the production of foundational cancer care components for future cancer facilities. The RMOH's and partners' investment into BCCOE was not only an investment towards a single health facility with a finite volume of patients, it was and continues to be a strategic investment into cancer care for the country and a potential model for others.

Author Contributions: Conceptualization, P.H.P., L.N.S., F.U. and L.R.H.; Methodology, P.H.P. and L.R.H.; Formal Analysis, P.H.P., C.S., L.N.S., F.U. and L.R.H.; Investigation, P.H.P.; Resources, F.U., L.N.S., L.B. and L.R.H.; Data Curation, P.H.P.; Writing-Original Draft Preparation, P.H.P.; WritingReview and Editing, P.H.P., F.K., N.G., C.R., J.M., A.C., L.L., L.B., L.N.S., J.M.M., F.U. and L.R.H.; Visualization, P.H.P. and L.R.H.; Supervision, L.R.H., F.U., L.N.S., L.B. and J.M.M.; Project Administration, P.H.P. All authors have read and agreed to the published version of the manuscript.

Funding: This research received no external funding.

Institutional Review Board Statement: Not applicable.

Informed Consent Statement: Not applicable.

Data Availability Statement: Not applicable.

Acknowledgments: The authors wish to acknowledge the frontline cancer workers, educators, and implementers in Rwanda whose hard work and commitment to cancer patients allows for ongoing growth of the national cancer program.

Conflicts of Interest: The authors declare no conflict of interest. 


\section{References}

1. Institute for Health Metrics and Evaluation (IHME). Global Burden of Disease Compare Data Visualization; IHME, University of Washington: Seattle, WA, USA, 2018.

2. Fitzmaurice, C.; Abate, D.; Abbasi, N.; Abbastabar, H.; Abd-Allah, F.; Abdel-Rahman, O.; Abdelalim, A.; Abdoli, A.; Abdollahpour, I.; et al.; Global Burden of Disease Cancer Collaboration. Global, Regional, and National Cancer Incidence, Mortality, Years of Life Lost, Years Lived With Disability, and Disability-Adjusted Life-Years for 29 Cancer Groups, 1990 to 2017: A Systematic Analysis for the Global Burden of Disease Study. JAMA Oncol. 2019, 5, 1749-1768. [CrossRef] [PubMed]

3. Ngoma, T. World Health Organization cancer priorities in developing countries. Ann. Oncol. 2006, 17, viii9-viii14. [CrossRef]

4. Stefan, D.C. Cancer Care in Africa: An Overview of Resources. J. Glob. Oncol. 2015, 1, 30-36. [CrossRef]

5. Theobald, S.; Brandes, N.; Gyapong, M.; El-Saharty, S.; Proctor, E.; Diaz, T.; Wanji, S.; Elloker, S.; Raven, J.; Elsey, H.; et al. Implementation research: New imperatives and opportunities in global health. Lancet 2018, 392, 2214-2228. [CrossRef]

6. McCarthy, E.A.; O'Brien, M.E.; Rodriguez, W.R. Training and HIV-Treatment Scale-Up: Establishing an Implementation Research Agenda. PLoS Med. 2006, 3, e304. [CrossRef] [PubMed]

7. National Institutes of Health. Implementation Science News, Resources and Funding for Global Health Researchers. Available online: https:/ / www.fic.nih.gov/ResearchTopics/Pages/ImplementationScience.aspx (accessed on 30 October 2019).

8. Aarons, G.A.; Hurlburt, M.; Horwitz, S.M. Advancing a Conceptual Model of Evidence-Based Practice Implementation in Public Service Sectors. Adm. Policy Ment. Heal. Ment. Health 2010, 38, 4-23. [CrossRef] [PubMed]

9. Moullin, J.C.; Dickson, K.S.; Stadnick, N.A.; Rabin, B.; Aarons, G.A. Systematic review of the Exploration, Preparation, Implementation, Sustainment (EPIS) framework. Implement. Sci. 2019, 14, 1-16. [CrossRef] [PubMed]

10. Tapela, N.M.; Tshisimogo, G.; Shatera, B.P.; Letsatsi, V.; Gaborone, M.; Madidimalo, T.; Ovberedjo, M.; Jibril, H.B.; Tsima, B.; Nkomazana, O.; et al. Integrating noncommunicable disease services into primary health care, Botswana. Bull. World Health Organ. 2019, 97, 142-153. [CrossRef] [PubMed]

11. Williams, J.R.; Blais, M.P.; Banks, D.; Dusablon, T.; Williams, W.O.; Hennessy, K.D. Predictors of the Decision to Adopt Motivational Interviewing in Community Health Settings. J. Behav. Health Serv. Res. 2013, 41, 294-307. [CrossRef]

12. Tapela, N.M.; Mpunga, T.; Hedt-Gauthier, B.; Moore, M.; Mpanumusingo, E.; Xu, M.J.; Nzayisenga, I.; Hategekimana, V.; Umuhizi, D.G.; Pace, L.E.; et al. Pursuing equity in cancer care: Implementation, challenges and preliminary findings of a public cancer referral center in rural Rwanda. BMC Cancer 2016, 16, 1-9. [CrossRef]

13. Stulac, S.; Binagwaho, A.; Tapela, N.M.; Wagner, C.M.; Muhimpundu, M.A.; Ngabo, F.; Nsanzimana, S.; Kayonde, L.; Bigirimana, J.B.; Lessard, A.J.; et al. Capacity building for oncology programmes in sub-Saharan Africa: The Rwanda experience. Lancet Oncol. 2015, 16, e405-e413. [CrossRef]

14. Shulman, L.N.; Mpunga, T.; Tapela, N.; Wagner, C.M.; Fadelu, T.; Binagwaho, A. Bringing cancer care to the poor: Experiences from Rwanda. Nat. Rev. Cancer 2014, 14, 815-821. [CrossRef]

15. Park, P.H.; Davey, S.; Fehr, A.E.; Butonzi, J.; Shyirambere, C.; Hategekimana, V.; Bigirimana, J.B.; Borg, R.; Uwizeye, R.; Tapela, N.; et al. Patient Characteristics, Early Outcomes, and Implementation Lessons of Cervical Cancer Treatment Services in Rural Rwanda. J. Glob. Oncol. 2018, 4, 1-11. [CrossRef]

16. Pace, L.E.; Dusengimana, J.M.V.; Shulman, L.N.; Schleimer, L.E.; Shyirambere, C.; Rusangwa, C.; Muvugabigwi, G.; Park, P.H.; Huang, C.; Bigirimana, J.B.; et al. Cluster Randomized Trial to Facilitate Breast Cancer Early Diagnosis in a Rural District of Rwanda. J. Glob. Oncol. 2019, 5, 1-13. [CrossRef]

17. Pace, L.E.; Dusengimana, J.-M.V.; Keating, N.L.; Hategekimana, V.; Rugema, V.; Bigirimana, J.B.; Costas-Chavarri, A.; Umwizera, A.; Park, P.H.; Shulman, L.N.; et al. Impact of Breast Cancer Early Detection Training on Rwandan Health Workers' Knowledge and Skills. J. Glob. Oncol. 2018, 4, 1-10. [CrossRef] [PubMed]

18. Shyirambere, C.; Xu, M.J.; Elmore, S.; Fadelu, T.; May, L.; Tapela, N.; Umuhizi, D.G.; Uwizeye, F.R.; Driscoll, C.; Muhayimana, C.; et al. Treating Nephroblastoma in Rwanda: Using International Society of Pediatric Oncology Guidelines in a Novel Oncologic Care Model. J. Glob. Oncol. 2016, 2, 105-113. [CrossRef]

19. Eberly, L.A.; Rusingiza, E.; Park, P.H.; Ngoga, G.; Dusabeyezu, S.; Mutabazi, F.; Harerimana, E.; Mucumbitsi, J.; Nyembo, P.F.; Borg, R.; et al. Nurse-Driven Echocardiography and Management of Heart Failure at District Hospitals in Rural Rwanda. Circ. Cardiovasc. Qual. Outcomes 2018, 11. [CrossRef]

20. Rich, M.L.; Miller, A.C.; Niyigena, P.; Franke, M.F.; Niyonzima, J.B.; Socci, A.; Drobac, P.C.; Hakizamungu, M.; Mayfield, A.; Ruhayisha, R.; et al. Excellent Clinical Outcomes and High Retention in Care Among Adults in a Community-Based HIV Treatment Program in Rural Rwanda. JAIDS J. Acquir. Immune Defic. Syndr. 2012, 59, e35-e42. [CrossRef]

21. Binagwaho, A.; Farmer, P.E.; Nsanzimana, S.; Karema, C.; Gasana, M.; Ngirabega, J.D.D.; Ngabo, F.; Wagner, C.M.; Nutt, C.T.; Nyatanyi, T.; et al. Rwanda 20 years on: Investing in life. Lancet 2014, 384, 371-375. [CrossRef]

22. Muhayimana, C.; Kennell-Heiling, S.; Svoboda, L.; Umuhizi, D.; Hategekimana, V.; Buswell, L. Oncology nursing workforce capacity building in rural Rwanda: Strengthening specialized cancer care through nursing education and skill development. Ann. Glob. Health 2016, 82, 484. [CrossRef]

23. Muvugabigwi, G.; Nshimiyimana, I.; Greenberg, L.; Hakizimana, E.; Ruhangaza, D.; Benewe, O.; Bhai, K.; Pepoon, J.R.; Fehr, A.E.; Park, P.H.; et al. Decreasing Histology Turnaround Time Through Stepwise Innovation and Capacity Building in Rwanda. J. Glob. Oncol. 2018, 4, 1-6. [CrossRef] [PubMed] 
24. Orozco, J.D.; Greenberg, L.A.; Desai, I.K.; Anglade, F.; Ruhangaza, D.; Johnson, M.; Ivers, L.; Milner, D.A.; Farmer, P.E. Building Laboratory Capacity to Strengthen Health Systems: The Partners In Health Experience. Clin. Lab. Med. 2018, 38, 101-117. [CrossRef]

25. Tuyishime, D. Kanombe Hospital Acquires Radiotherapy Machine for Cancer Treatment; KT Press: Kigali, Rwanda, 2017.

26. Forman, L. What contribution have human rights approaches made to reducing AIDS-related vulnerability in sub-Saharan Africa? Glob. Heal. Promot. 2013, 20, 57-63. [CrossRef]

27. Mwaura, G. Good News for Rwanda's Breast Cancer and Diabetes Patients. The New Times, 10 December 2016.

28. Tapela, N.; Nzayisenga, I.; Sethi, R.; Bigirimana, J.B.; Habineza, H.; Hategekimana, V.; Mantini, N.; Mpunga, T.; Shulman, L.N.; Lehmann, L. Treatment of Chronic Myeloid Leukemia in Rural Rwanda: Promising Early Outcomes. J. Glob. Oncol. 2016, 2, 129-137. [CrossRef]

29. Neal, C.; Rusangwa, C.; Borg, R.; Mugunga, J.C.; Kennell-Heiling, S.; Shyirambere, C.; Pritchett, N.; Muhayimana, C.; Ntakirutimana, E.; Tapela, N.; et al. Cost of Treating Pediatric Cancer at the Butaro Cancer Center of Excellence in Rwanda. J. Glob. Oncol. 2018, 4, 1-7. [CrossRef]

30. Neal, C.; Rusangwa, C.; Borg, R.; Tapela, N.; Mugunga, J.C.; Pritchett, N.; Shyirambere, C.; Ntakirutimana, E.; Park, P.H.; Shulman, L.N.; et al. Cost of Providing Quality Cancer Care at the Butaro Cancer Center of Excellence in Rwanda. J. Glob. Oncol. 2018, 4, 1-7. [CrossRef]

31. Pace, L.E.; Mpunga, T.; Hategekimana, V.; Dusengimana, J.V.; Habineza, H.; Bigirimana, J.B.; Mutumbira, C.; Mpanumusingo, E.; Ngiruwera, J.P.; Tapela, N.; et al. Delays in Breast Cancer Presentation and Diagnosis at Two Rural Cancer Referral Centers in Rwanda. Oncologist 2015, 20,780-788. [CrossRef]

32. Mitema, A.; Maree, L.; Young, A. Cancer treatment in Africa: The importance of the role of nursing. ecancermedicalscience 2019, 13, 944. [CrossRef] [PubMed]

33. Simms, K.T.; Steinberg, J.; Caruana, M.; Smith, M.A.; Lew, J.-B.; Soerjomataram, I.; Castle, P.E.; Bray, F.; Canfell, K. Impact of scaled up human papillomavirus vaccination and cervical screening and the potential for global elimination of cervical cancer in 181 countries, 2020-2099: A modelling study. Lancet Oncol. 2019, 20, 394-407. [CrossRef]

34. Rubagumya, F.; Costas-Chavarri, A.; Manirakiza, A.; Murenzi, G.; Uwinkindi, F.; Ntizimira, C.; Rukundo, I.; Mugenzi, P.; Rugwizangoga, B.; Shyirambere, C.; et al. State of Cancer Control in Rwanda: Past, Present, and Future Opportunities. JCO Glob. Oncol. 2020, 6, 1171-1177. [CrossRef] [PubMed]

35. Rubagumya, F.; Greenberg, L.; Manirakiza, A.; DeBoer, R.; Park, P.H.; Mpunga, T.; Shulman, L.N. Increasing global access to cancer care: Models of care with non-oncologists as primary providers. Lancet Oncol. 2017, 18, 1000-1002. [CrossRef]

36. Akwagyiram, A. How can Africa move away from aid dependence? BBC News, 1 May 2013.

37. Herbert, B. In America; Refusing To Save Africans. The New York Times, 11 June 2001; 17.

38. Engebretsen, E.; Heggen, K.; Das, S.; Farmer, P.; Ottersen, O.P. Paradoxes of sustainability with consequences for health. Lancet Glob. Health 2016, 4, e225-e226. [CrossRef]

39. El-Sadr, W.M.; Holmes, C.B.; Mugyenyi, P.; Thirumurthy, H.; Ellerbrock, T.; Ferris, R.; Sanne, I.; Asiimwe, A.; Hirnschall, G.; Nkambule, R.N.; et al. Scale-up of HIV Treatment Through PEPFAR: A historic public health achievement. JAIDS J. Acquir. Immune Defic. Syndr. 2012, 60, S96-S104. [CrossRef] [PubMed] 